\title{
Cronología del desarrollo puberal en niñas escolares de Santiago: relación con nivel socio-económico e índice de masa corporal
}

\author{
Ethel Codner D, N ancy U nanue M, Ximena G aete V, \\ Alvaro Barrera P, Dennis Mook-Kanamori ${ }^{\text {b }}$, \\ Rodrigo Bazaes C, Alejandra Avila Aa, Fernando Cassorla G. \\ Age of pubertal events in Chilean \\ school age girls and its relationship \\ with socioeconomic status and body \\ mass index
}

Background: Recent studies in the United States have demonstrated that a significant proportion of girls show thelarche before the age of eight years. Nutritional status, geographic influences and racial factors are known to affect the timing of puberty. Aim: To evaluate the age of onset of puberty, development of secondary sexual characteristics and menarche in Chilean girls, and its relation to obesity and socioeconomic status. Material and methods: Healthy girls attending elementary school, from first to ninth grade in Santiago, Chile, were studied. A pediatric endocrinologist evaluated pubertal development using Tanner classification. Breast development was assessed by inspection and breast palpation. Average age of onset of pubertal events was determined by probit analysis. Results: A total of 758 girls, aged 5.8 to 16.1 years, were recruited. Obesity, defined as a BMI greater than 90th percentile, was found in $24.4 \%$. The age of menarche was 12.7 years, the onset of Tanner stage 2 breast development and pubic hair was at 8.9 and 10.4 years, respectively. Sixteen percent of girls aged 7 to 7,9 years, had thelarche. Upper class girls showed a later onset of breast Tanner stage 4 stage than low-middle class girls. Obesity was not found in logistic regression analysis to be a significant predictive factor in the onset of puberty. Conclusions: The age of menarche has not changed in the last thirty years, but an earlier onset of thelarche has occurred. The high frequency of thelarche between 7 and 8 years suggests that the normal age of breast development should be revised (Rev Méd Chile 2004; 132: 801-8).

(Key Words: Body mass index; Menarche; Puberty; Sex maturation)

\footnotetext{
Recibido el 10 de diciembre, 2003. Aceptado el 29 de abril, 2004.

${ }^{1}$ Instituto de Investigaciones Materno Infantil (IDIMI), Facultad de Medicina, Universidad de Chile y Hospital Clínico San Borja Arriarán, Servicio de Salud Metropolitano Centro. aEnfermera Universitaria.

bAlumno de la Carrera de Medicina de la Universidad de Chile.
}

Correspondencia a: Dra. Ethel Codner D. IDIMI, Casilla 
$\mathrm{L}_{\mathrm{t}}^{\mathrm{a}}$ a pubertad es el proceso biológico que permite adquirir la capacidad reproductiva. La edad de inicio del desarrollo puberal y de la menarquia han presentado una tendencia secular a ocurrir más precozmente ${ }^{1}$. El primer trabajo realizado en Chile sobre el tema fue publicado en el año $1888^{2}$, describiendo una edad de la menarquia de 16 años en adolescentes de Santiago.

Los estudios clásicos de edad de los distintos eventos puberales fueron efectuados en Inglaterra por Marshall y Tanner, que evaluaron, en forma prospectiva, a 192 niños desde los 8 años ${ }^{3}$. Este estudio, realizado en niñas institucionalizadas de bajo nivel socio-económico, determinó que la edad promedio de inicio de la pubertad en niñas era de 10,5 años. Recientemente, HermanGiddens evaluó a 17.077 niñas de 3 a 12 años de Estados Unidos de América (USA) y determinó que el $5 \%$ de las niñas blancas y $15 \%$ de las afroamericanas, entre 7 y 8 años, presentaban desarrollo mamario ${ }^{4}$, y que la edad de inicio de la pubertad se habría adelantado a los 9,96 años en población caucásica y a los 8,86 años en afroamericanas. Estos datos fueron recientemente corroborados por el estudio americano NHANES III ${ }^{5}$.

La edad de inicio de la pubertad es determinada por factores genéticos ${ }^{6,7}$, ambientales ${ }^{1}$ y étni$\cos ^{4,8-10}$. Por ejemplo, los norteamericanos de origen mexicano serían más precoces que los americanos caucásicos ${ }^{8}$. Nuestro grupo, recientemente, estudió a 131 niñas eutróficas de 6 a 10 años, de nivel socio-económico medio y bajo, demostrando que un porcentaje importante de las niñas de 7 a 8 años presentan desarrollo mamario ${ }^{11}$. Con la hipótesis de que existe un adelantamiento en la edad en que ocurre la aparición de los caracteres sexuales, y que el sobrepeso y el nivel socio-económico son elementos que influyen en dicho fenómeno, decidimos ampliar dicho estudio a niñas de 5 a 17 años, incluyendo las niñas con sobrepeso, de colegios públicos y privados de Santiago.

\section{MATERIAL y MÉTOdOS}

Se realizó un estudio transversal de niñas escolares de Santiago provenientes de 7 colegios públicos del sector sur y centro de Santiago y dos colegios privados del área oriente de Santiago, elegidos en forma aleatoria. Las niñas fueron reclutadas entre el año 2000 y 2003. Un endocrinólogo infantil (EC, UN o XG) evaluó el desarrollo puberal según la clasificación de Tanner. El desarrollo mamario se etapificó usando inspección y palpación mamaria. El peso se determinó en una balanza convencional con precisión de 100 $g$, la talla fue obtenida en un estadiómetro de pared convencional. La antropometría se analizó según la curva NCHS $2000^{12}$, y los resultados se presentan como desviación estándar (DE) para la edad (puntaje Z). Se definió obesidad como un índice de masa corporal (IMC) mayor o igual al percentil 90 de la curva NCHS $2000^{12}$. Todas las pacientes fueron interrogadas por la presencia de alguna patología crónica, y se excluyeron aquellas que informaron enfermedades renales, gastrointestinales y cardiopulmonares.

El estudio fue aprobado por el Comité de Ética de Pediatría del Hospital San Borja Arriarán. A través de sus respectivos colegios, se envió consentimiento informado a los padres de las niñas de primero básico a noveno año de educación escolar, y sólo fueron examinadas las niñas cuyos padres dieron autorización por escrito.

Se determinó la edad de aparición de los eventos puberales según análisis de próbito, y se analizaron las diferencias en la edad de inicio de la pubertad entre colegios públicos y privados por el método de spotencia relativa mediana»según lo descrito por Robertson y Preisler ${ }^{13}$. Los resultados obtenidos se compararon con los obtenidos por Herman-Giddens ${ }^{4}$. Para los análisis de medidas antropométricas se utilizó la prueba t de Student 0 ANOVA, según el caso. Finalmente, se realizó un análisis de regresión logística binaria para determinar el efecto del IMC sobre la presencia de desarrollo puberal. Se muestran los resultados como promedio \pm error estándar (EE). Se consideró como significativo un $\mathrm{p}<0,05$.

\section{Resultados}

Se estudiaron 758 niñas escolares, 605 de ellas provenientes de colegios públicos y las restantes de colegios privados. La edad promedio fue de

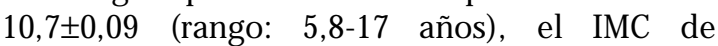
$0,60 \pm 0,04 \mathrm{DE}, \mathrm{y}$ el $\mathrm{Z}$ score de talla fue de $-0,03 \pm 0,04$. El $24,4 \%$ de las niñas presentó un IMC 
Tabla 1. Características antropométricas y distribución por edad

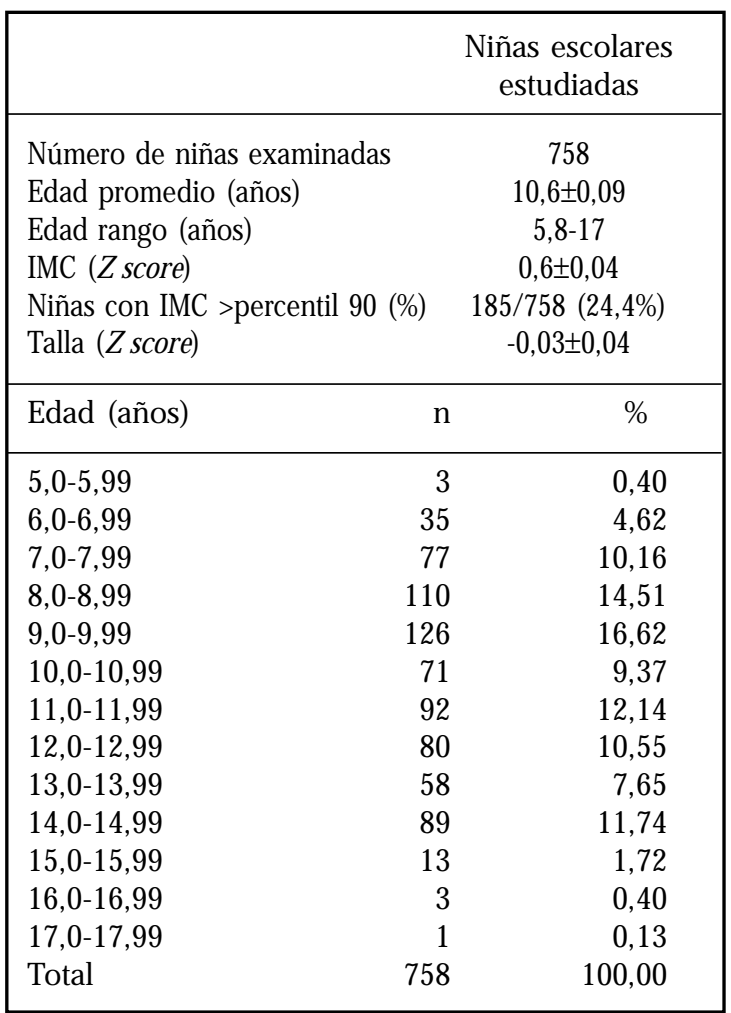

Z score: Desviación estándar para la edad según las curvas NCHS 2000. IMC: índice de masa corporal. mayor al percentil 90. Las características clínicas y distribución por edad se detallan en la Tabla 1.

La edad promedio en alcanzar el estadio Tanner 2 de mamas fue de 8,88 $\pm 0,08$ años, y de 10,36 $\pm 0,51$ años para el estadio Tanner 2 de vello púbico. Ciento noventa y dos niñas ya habían presentado la menarquia, la que fue a los 12,68 $\pm 0,09$ años. Las edades de aparición de las diferentes etapas de desarrollo puberal, comparado con el estudio de Herman-Giddens, se presentan en la Tabla 2.

En las niñas menores de 6 años no se detectaron casos de telarquia ni pubarquia. En las niñas de 6 a 6,99 años se detectó un caso de pubarquia aislada (2,8\%). En el grupo de niñas de 7 a 7,99 años sólo una niña presentó vello púbico $(1,3 \%)$, pero se detectaron 13 niñas $(16,9 \%)$ con desarrollo mamario. De estas 13 niñas, 11 tenían entre 7,5 y 7,99 años, lo que representa a $25 \%$ de las niñas de esta edad, pero ninguna presentó simultáneamente vello púbico. En las niñas de 7 a 7,49 sólo $2(6,1 \%)$ tuvieron telarquia. Las niñas de menor edad que mostraron simultáneamente desarrollo de vello púbico y mamario tenían entre 8 a 8,99 años y correspondieron a $2,7 \%$ de este grupo. En la Figura 1 se detalla el porcentaje de niñas con desarrollo mamario y púbico para cada edad.

Tabla 2. Edad promedio de transición (años \pm error estándar) a los diferentes estadios de desarrollo puberal en el grupo de escolares de Santiago (ID IM I) y en los escolares norteamericanos caucásicos y afroamericanos

\begin{tabular}{|lcccc|}
\hline & & IDIMI (años) & $\begin{array}{c}\text { Herman-Giddens } \\
\text { población } \\
\text { caucásica (años) } \dagger\end{array}$ & $\begin{array}{c}\text { Herman-Giddens } \\
\text { población afro- } \\
\text { americana (años) } \dagger\end{array}$ \\
\hline Desarrollo mamario (estadio de Tanner) & 2 & $8,88 \pm 0,08$ & $9,96 \pm 0,03$ & $8,87 \pm 0,09$ \\
& 3 & $10,42 \pm 0,11$ & $11,30 \pm 0,03$ & $10,19 \pm 0,08$ \\
& 4 & $11,82 \pm 0,08$ & $\mathrm{n} / \mathrm{i}$ & $\mathrm{n} / \mathrm{i}$ \\
Vello púbico (estadio de Tanner) & 5 & $12,87 \pm 0,11$ & $\mathrm{n} / \mathrm{i}$ & $\mathrm{n} / \mathrm{i}$ \\
& 2 & $10,36 \pm 0,51$ & $10,51 \pm 0,03$ & $8,78 \pm 0,03$ \\
& 3 & $11,27 \pm 0,08$ & $11,53 \pm 0,03$ & $10,35 \pm 0,09$ \\
Menarquia & 4 & $12,09 \pm 0,08$ & $\mathrm{n} / \mathrm{i}$ & $\mathrm{n} / \mathrm{i}$ \\
& 5 & $13,10 \pm 0,11$ & $\mathrm{n} / \mathrm{i}$ & $\mathrm{n} / \mathrm{i}$ \\
& & $12,680,09$ & $12,88 \quad 0,06$ & $12,16 \quad 0,11$ \\
\hline
\end{tabular}

n/i: No informado. †: Herman-Giddens ME, Slora EJ, Wasserman RC, Bourdony CJ, Bhapkar MV, Koch GG et al. Secondary sexual characteristics and menses in young girls seen in office practice: a study from the Pediatric Research in Office Settings network. Pediatrics 1997; 99: 505-124. 
La comparación de las niñas de colegios privados con los públicos se detalla en la Tabla 3. El IMC fue significativamente mayor en los colegios públicos (puntaje Z 0,70 $\pm 0,04$ vs $0,30 \pm 0,07$, p
$<0,00001$ ), así como también fue mayor el porcentaje de obesidad $(27,7 \%$ vs $11,1 \%, p<0,0001)$. El estadio de Tanner 4 de mama ocurrió significativamente más tarde en el nivel socio-económico alto

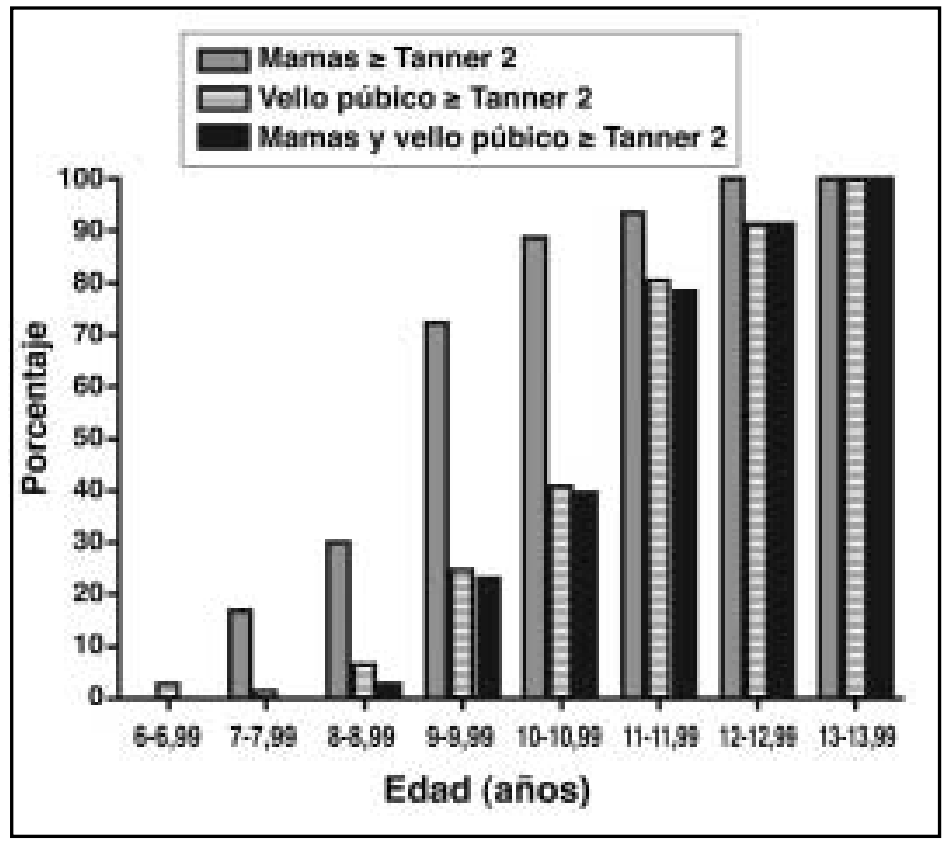

Figura 1. Prevalencia de desarrollo mamario o pubiano en estadio $\geq$ Tanner 2 por edad.

Tabla 3. Antropometría y edad de aparición de los eventos puberales en las niñas de colegios públicos y colegios privados de Santiago

\begin{tabular}{|c|c|c|}
\hline & Público & Privado \\
\hline $\mathrm{n}$ & 605 & 153 \\
\hline Edad (años) & $10,89 \pm 0,1$ & $9,64 \pm 0,13^{* 4 *}$ \\
\hline IMC (kg/mt2) & $0,7 \pm 0,04$ & $0,3 \pm 0,07^{* * 1 *}$ \\
\hline Niñas con IMC >percentil $90(\%)$ & $168(27,7)$ & $17(11,1)^{* * * *}$ \\
\hline \multicolumn{3}{|c|}{ Desarrollo mamario (estadio de Tanner) } \\
\hline 2 (años) & $8,94 \pm 0,10$ & $8,75 \pm 0,15$ \\
\hline 3 (años) & $10,36 \pm 0,12$ & $10,64 \pm 0,23$ \\
\hline 4 (años) & $11,74 \pm 0,09$ & $12,24 \pm 0,23 *$ \\
\hline 5 (años) & $12,84 \pm 0,11$ & $13,14 \pm 0,34$ \\
\hline \multicolumn{3}{|c|}{ Desarrollo de vello púbico (estadio de Tanner) } \\
\hline 2 (años) & $10,46 \pm 0,16$ & $10,47 \pm 0,29$ \\
\hline 3 (años) & $11,35 \pm 0,09$ & $11,26 \pm 0,18$ \\
\hline 4 (años) & $12,10 \pm 0,09$ & $12,34 \pm 0,21$ \\
\hline 5 (años) & $13,13 \pm 0,11$ & $13,38 \pm 0,33$ \\
\hline
\end{tabular}

$*$ : $\mathrm{p} \leq 0,05 . * * * \mathrm{p}<0,001$. 
$(12,34 \pm 0,21$ versus $12,1 \pm 0,09$ años, $p \leq 0,05)$. El estadio de Tanner 3 y 5 también mostraron una tendencia a ocurrir más tardíamente en las niñas de colegios de nivel socio-económico alto, pero esto no alcanzó significación estadística. No existió diferencia en la edad de desarrollo de vello púbico. Sólo 8 niñas de colegios privados ya habían tenido su menarquia, razón por la que no es posible analizar la edad de menarquia por tipo de colegio.

Las niñas con y sin desarrollo puberal mostraron un Z score de IMC semejante, luego de ajustar por edad. El análisis de regresión logística binaria, no mostró que el IMC sea un determinante significativo en el inicio del desarrollo puberal en esta población de niñas chilenas $(\Omega=0,119$; $p=0,37$ ).

\section{DisCUSIÓN}

La tendencia secular descrita para los países europeos demuestra un adelanto progresivo de la menarquia desde mediados del siglo XIX hasta la década 1960-69, en el siglo XX11. Posteriormente, la edad de la menarquia en los países del hemisferio norte se ha mantenido estable desde 1970-791,8. En Chile, en 1888 la doctora Eloísa Díaz, la primera mujer médico chilena, describió que la edad más frecuente de la menarquia en Santiago era a los 16 años $^{2}$. En 1974, Rona informa una edad de menarquia de 12,6 años en un grupo de 354 niñas del área norte de Santiago ${ }^{14}$. Este estudio, efectuado en 758 escolares de Santiago, demostró una edad de menarquia de 12,68 años, semejante a lo descrito en la población americana por Herman-Giddens ${ }^{4}$. Por lo tanto, la edad de la menarquia en Chile, al menos en el grupo socio-económico bajo, ha mostrado un comportamiento secular semejante al de los países europeos ${ }^{1}$, con un adelanto progresivo desde fines del siglo XIX hasta los años setenta del siglo XX, momento desde el que no ha mostrado modificaciones relevantes. Nuestro trabajo previo no evaluó la edad de la menarquia ya que sólo se estudiaron niñas de 6 a 10 años ${ }^{11}$.

La población estudiada por nosotros presenta su telarquia a los 8,9 años, un año antes que la población americana caucásica y a una edad semejante a la población afroamericana ${ }^{4}$. El año 1988 Burrows et al, publican una serie chilena de niñas de 8 a 15 años, en que la edad de inicio de la pubertad correspondió a 10,5 años ${ }^{15}$. Estos datos sugieren que la telarquia en niñas chilenas está ocurriendo más precozmente que hace una década, pero que la progresión de los eventos puberales es lenta ya que la menarquia ocurre aproximadamente cuatro años después que la telarquia. Estudios longitudinales han demostrado que existe una relación inversa entre edad de inicio y duración de la pubertad ${ }^{16}$. En los niños, en cambio, no se ha detectado un adelanto de la pubertad en Chile ${ }^{11}$.

El hecho que nuestras niñas escolares sean más precoces que la población americana caucásica había sido publicado previamente por Valenzuela, en el año 1983, quién comparó a los escolares chilenos con los de otros países y concluyó que el crecimiento prepuberal, estirón puberal, finalización del crecimiento y aparición de caracteres sexuales en las niñas era más precoz que en niñas francesas e inglesas ${ }^{17}$.

El porcentaje de niñas con desarrollo mamario en cada grupo etáreo fue mayor en niñas chilenas que en niñas americanas caucásicas en todas las edades, pero semejante a las afroamericanas. El $16,4 \%$ de las niñas chilenas de Santiago presentan telarquia entre los 7 y 7,99 años, las cuales tenían mayoritariamente entre los 7,5 y 7,99 años, y no presentaron simultáneamente vello púbico, sugiriendo que se puede tratar de casos de telarquia aislada 0 de cuadros de pubertad lentamente progresiva. El hecho que las niñas tengan telarquia aislada sin vello púbico, ha sido descrito en otras poblaciones, asociado a un aumento del IMC y porcentaje de grasa corporal total ${ }^{18}$, aunque también puede tener un componente genético, ya descrito en el nivel socio-económico medio y bajo chileno ${ }^{15}$. En el tramo de 8 a 8,99 años $27 \%$ tenía telarquia, pero sólo $3 \%$ tenía desarrollo de mamas y vello pubiano simultáneo. Estos porcentajes nuevamente son mayores que los reportados por Burrows en $1988^{15}$.

Existió una tendencia a un desarrollo mamario más tardío en el nivel socio-económico alto (para Tanner 4 fue estadísticamente significativo), elemento que ya se había reportado anteriormente en población chilena ${ }^{15}$. Estudios en múltiples 
países han demostrado diferencias en la edad de los eventos puberales según las condiciones sociales del grupo estudiado ${ }^{1}$.

El avance en la edad de desarrollo puberal en niñas se ha asociado con un aumento de la prevalencia de la obesidad. El grado de adiposidad es el elemento más importante en adelantar la madurez biológica en niñas sanas, y por lo tanto acelera la edad ósea ${ }^{19}$. Las niñas con menarquia adelantada, o una talla mayor para su edad, tienen mayor peso que las niñas en que estos eventos ocurren a una edad promedio ${ }^{9,20}$. Incluso una adiposidad aumentada a los 5 años es un factor pronóstico de adelanto en la pubertad posteriormente ${ }^{21}$. El tejido adiposo tiene importancia para adquirir y mantener la capacidad reproductiva, existiendo un tamaño corporal crítico para desencadenar la pubertad, presentar la menarquia y mantener los ciclos menstruales ${ }^{22-24}$. Esto queda ejemplificado en los enfermos con anorexia nerviosa o temor a la obesidad que presentan amenorrea o retraso puberal, retrospectivamente ${ }^{25,26}$. En el estudio de Herman-Giddens las niñas con desarrollo mamario o de vello púbico tenían un mayor IMC $^{32}$. Nuestro grupo presentó $24,4 \%$ de sobrepeso, en cambio la población caucásica presentó $14 \%$, y $21-28 \%$ en el grupo afroamericano ${ }^{34}$. Los resultados obtenidos en nuestra población son semejantes a los obtenidos en el grupo afroamericano, que presenta telarquia más adelantada, mayor sobrepeso que la caucásica y pérdida de la asociación estadística entre el sobrepeso y desarrollo puberal ${ }^{32}$. Es posible que la evaluación de la composición corporal con métodos tales como impedanciometría 0 medición de pliegues, nos hubiese permitido evaluar con mayor exactitud el efecto de la adiposidad sobre el adelanto de la pubertad. Rona y Pereira demostraron en niñas chilenas una pubertad más adelantada, en asociación con aumento de los pliegues corporales ${ }^{14}$.

Una de las limitaciones de nuestro trabajo es que los colegios fueron seleccionados al azar. No podemos asegurar que realmente correspondan a una muestra representativa de Santiago, pero por otro lado, el estudio tiene la fortaleza de que los datos provienen de población escolar sana, eliminando la selección de pacientes con patologías que se produce al evaluar niñas que acuden a una consulta pediátrica ${ }^{4}$.

El manejo de las pacientes con desarrollo mamario antes de los 8 años ha sido motivo de controversia en los últimos años. La Lawson Wilkins Endocrine Society recomienda el estudio de las pacientes con pubertad precoz de 7 a 8 años de edad sólo si existe compromiso del pronóstico de talla, problemas psicológicos o elementos sugerentes de patología tumoral del sistema nervioso central ${ }^{34}$. Incluso se ha recomendado sólo estudiar a las niñas menores de 7 años $^{35}$. En cambio, recientemente un panel de expertos chilenos recomienda el estudio de todas las pacientes menores de 8 años con pubertad precoz ${ }^{36}$. En vista de la alta frecuencia de telarquia entre los 7 y 8 años encontrada en este trabajo, planteamos que los casos de telarquia sin vello púbico podrían corresponder a variantes normales del desarrollo. A pesar de que estas pacientes podrían manejarse conservadoramente con la observación clínica, en ellas se ha descrito un aumento de la resistencia insulínica ${ }^{37}$, ciertos problemas conductuales transitorios, trastornos psicosomáti$\cos ^{38,39}$ y aumento del riesgo de cancer de mama en la población genéticamente predispuesta 40 .

Concluimos que en el grupo de niñas evaluadas la edad de aparición del botón mamario se ha adelantado, que es frecuente la aparición de telarquia antes de los ocho años, pero que la edad de la menarquia se ha mantenido estable en los últimos años. Es posible que factores genéticos, raciales, nutricionales, geográficos y ambientales determinen que el desarrollo puberal en la población chilena esté ocurriendo tempranamente. 


\section{REFERENCIAS}

1. Parent as, Tellmann G, Juul A, Skakkebaek NE, TOPPARI J, Bourguignon JP. The timing of normal puberty and the age limits of sexual precocity: variations around the world, secular trends, and changes after migration. Endocr Rev 2003; 24: 668-93.

2. DíAZ E. Breves observaciones sobre la aparición de la pubertad en la mujer chilena i de las predisposiciones patolójicas propias del sexo. Rev Méd Chile 1888; 16: 289-98, 337-46.

3. MARShall WA, TANNER JM. Variations in pattern of pubertal changes in girls. Arch Dis Child 1969; 44: 291-303.

4. Herman-Giddens ME, Slora EJ, Wasserman RC, BOURDONY CJ, BHAPKAR MV, Koch GG ET AL. Secondary sexual characteristics and menses in young girls seen in office practice: a study from the Pediatric Research in Office Settings network Pediatrics 1997; 99: 505-12.

5. NHANES III. NHANES III Reference manuals and reports (CD-ROM). Analytic and reporting guidelines: the Third National Health and Nutrition Examination Survey (1988-94). National Center for Health Statistics, Centers for Disease Control and Prevention, Hyattsville, MD., 1997

6. Meyer JM, Eaves LJ, Heath AC, Martin NG. Estimating genetic influences on the age at menarche: a survival analysis approach. Am J Med Genet 1991; 39: 148-54.

7. TReloar SA, Martin NG. Age at menarche as a fitness trait: nonadditive genetic variance detected in a large twin sample. Am J Hum Genet 1990; 47: 137-48.

8. Chumea WC, Schubert CM, Roche AF, Kulin HE, LeE PA, Himes JH et AL. Age at menarche and racial comparisons in US girls. Pediatrics 2003; 111: 110-3.

9. Anderson SE, Dallal GE, Must A. Relative weight and race influence average age at menarche: results from two nationally representative surveys of US girls studied 25 years apart. Pediatrics 2003; 111: 844-50.

10. Sun SS, Schubert CM, Chumiea WC, Roche AF, KuLiN HE, LeE PA ET AL. National estimates of the timing of sexual maturation and racial differences among US children. Pediatrics 2002; 110: 911-9.
11. Gaete X, Unanue N, Avila A, Cassoria F. Cambios en la edad de inicio de la pubertad en niñas de la comuna de Santiago: Implicancias para el diagnóstico de la pubertad precoz. Rev Chil Pediatr 2002; 73: 363-8.

12. Ogden CL, Kuczmarski RJ, Flegal KM, Mei Z, Guo S, Wei R et aL. Centers for Disease Control and Prevention 2000 growth charts for the United States: improvements to the 1977 National Center for Health Statistics version. Pediatrics 2002; 109: 45-60.

13. Robertson JL, Preisier HK. 1992 Pesticide Bioassays with Arthropods. CRC Press.

14. Rona R, Pereira G. Factors that influence age of menarche in girls in Santiago, Chile. Hum Biol 1974; 46: 33-42.

15. Burrows R, Leiva L, Mauricci A, Zvaighaft A, Muzzo S. Características de la pubertad de niñas escolares de la Región Metropolitana. Rev Chil Pediatr 1988; 59: 21-5.

16. Marti-Henneberg C, Vizmanos B. The duration of puberty in girls is related to the timing of its onset. J Pediatr 1997; 131: 618-21.

17. ValenZuela CY. Pubertal origin of the larger sex dimorphism for adult stature of a Chilean population. Am J Phys Anthropol 1983; 60: 53-60.

18. Biro FM, Lucky AW, Simbartl LA, Barton BA, Daniels SR, Striegel-Moore R et al. Pubertal maturation in girls and the relationship to anthropometric changes: pathways through puberty. J Pediatr 2003; 142: 643-6.

19. Russell D, Keil M, Bonat S, Uwaifo G, Nicholson J, McDufFIE J ET AL. The relation between skeletal maturation and adiposity in African American and Caucasian children. J Pediatr 2001; 139: 844-8.

20. Biro FM, McMahon RP, Striegel-Moore $R$, Crawford PB, Obarzanek E, Morrison JA et al. Impact of timing of pubertal maturation on growth in black and white female adolescents: The National Heart, Lung, and Blood Institute Growth and Health Study. J Pediatr 2001; 138: 636-43.

21. Davison KK, Susman EJ, Birch L. Percent body fat at age 5 predicts earlier pubertal development among girls at age 9 . Pediatrics 2003; 111: 815-21.

22. Frisch RE, McARthuR JW. Menstrual cycles: fatness as a determinant of minimum weight for height necessary for their maintenance or onset. Science 1974; 185: 949-51. 
23. Frisch RE, REveue R. Height and weight at menarche and a hypothesis of critical body weight and adolescent events. Science 1970; 169: 397-9.

24. Frisch RE, Reveue R. Height and weight at menarche and a hypothesis of menarche. Arch Dis Child 1971; 46: 695-701.

25. Munoz MT, Argente J. Anorexia nervosa in female adolescents: endocrine and bone mineral density disturbances. Eur J Endocrinol 2002; 147: 275-86.

26. Pugliese MT, Lifshitz F, Grad G, Fort P, MarksKatz M. Fear of obesity. A cause of short stature and delayed puberty. N Engl J Med 1983; 309: 513-8.

27. Terasawa E, Fernández DL Neurobiological mechanisms of the onset of puberty in primates. Endocr Rev 2001; 22: 111-51.

28. Сненав FF, Mounzih K, Lu R, Lim ME. Early onset of reproductive function in normal female mice treated with leptin. Science 1997; 275: 88-90.

29. Mantzoros CS, Flier JS, Rogol AD. A longitudinal assessment of hormonal and physical alterations during normal puberty in boys. V. Rising leptin levels may signal the onset of puberty. J Clin Endocrinol Metab 1997; 82: 1066-70.

30. Roemmich JN, Rogol AD. Role of leptin during childhood growth and development. Endocrinol Metab Clin North Am 1999; 28: 749-64, viii.

31. Horlick MB, Rosenbaum M, Nicolson M, Levine LS, Fedun B, Wang J et al. Effect of puberty on the relationship between circulating leptin and body composition. J Clin Endocrinol Metab 2000; 85: 2509-18.

32. Kaplowitz PB, Slora EJ, Wasserman RC, Pedlow SE, HERMAN-GIDDENS ME. Earlier onset of puberty in girls: relation to increased body mass index and race. Pediatrics 2001; 108: 347-53.
33. WANG Y. Is obesity associated with early sexual maturation? A comparison of the association in American boys versus girls. Pediatrics 2002; 110: 903-10.

34. KapLowitz PB, ObeRFIEID SE. Reexamination of the age limit for defining when puberty is precocious in girls in the United States: implications for evaluation and treatment. Drug and Therapeutics and Executive Committees of the Lawson Wilkins Pediatric Endocrine Society. Pediatrics 1999; 104: 936-41.

35. Elders MJ, Scott CR, Frindik JP, Kemp SF. Clinical workup for precocious puberty. Lancet 1997; 350: 457-8.

36. García $H$, Youlton R, Burrows R, Cattann A. Consenso sobre el diagnóstico y tratamiento de la pubertad precoz central. Rev Méd Chile 2003; 131: 95-110.

37. MidyetT LK, Moore WV, Jacobson JD. Are pubertal changes in girls before age 8 benign? Pediatrics 2003; 111: 47-51.

38. Sonis WA, Comite F, Blue J, Pescovitz OH, Rahn CW, HeNCH KD ET AL. Behavior problems and social competence in girls with true precocious puberty. J Pediatr 1985; 106: 156-60.

39. Ehrhardt AA, Meyer-Bahlburg HF, Beil JJ, Cohen SF, Healey JM, Stiel R et al. Idiopathic precocious puberty in girls: psychiatric follow-up in adolescence. J Am Acad Child Psychiatry 1984; 23: 2333.

40. Hamiton AS, Mack TM. Puberty and genetic susceptibility to breast cancer in a case-control study in twins. N Engl J Med 2003; 348: 2313-22.

Agradecimientos

Queremos agradecer a todas las alumnas que aceptaron ser examinadas y a los colegios que permitieron hacerlo. 